\title{
Note on Topics of regions separated by boundaries.
}

\author{
By Dr R. F. Moirhtad, Glasgow.
}

(Received 20th May 1925. Read 5th June 1925.)

\section{I.}

$S_{r}$ denotes an infinite space of $r$ dimensions. Such a space is divided into two regions by a $S_{r-1}$. An infinite line is divided into $n+1$ regions by $n$ points. An infinite plane or other surface topically equivalent to it is divided into two regions by an infinite line.

Two infinite lines intersecting in one point divide the $S_{2}$ into $2+2$ regions.

A third infinite line intersecting each of the other two in one point is itself divided into three parts, each of which separates one of the previous regions into two regions.

Thus three lines of which each pair has but one intersection divides $S_{2}$ into $2+2+3$ regions.

Continuing this reasoning we see that $n$ such infinite lines divide $S_{2}$ into $2+2+3+\ldots+n$ regions. Thus if $\phi_{r}(n)$ denotes the number of regions into which a $S_{r}$ is divided by $n S_{r-1}$ 's, each pair of which have but one intersection in a $S_{r m, 2}$ we have

$$
\text { * } \phi_{2}(n)=1+1+2+3+\ldots+n=1+\frac{n . n+1}{2}=\frac{1}{2}\left(n^{2}+n+2\right)
$$

$={ }^{n} C_{2}+{ }^{n} C_{1}+1$ when ${ }^{n} C_{r}$ is the number of combinations of $n$ things taken $r$ at a time.

Thus $\phi_{2}(n-1)=1+{ }^{n-1} C_{1}+{ }^{n-1} C_{2}$

By similar reasoning we find that if a $S_{2}$ contains $n S_{2}^{\prime}$ 's each pair of which intersect one another in a $S_{1}$ and no three of which have a common $S_{1}$ and no four of which have a common point,

$$
\begin{aligned}
\phi_{3}(n)= & 2+\phi_{2}(1)+\phi_{2}(2)+\ldots+\phi_{2}(n-1) \\
= & 1+1+\left(1+{ }^{2} C_{1}\right)+\left(1+{ }^{2} C_{1}+{ }^{2} C_{2}\right)+\left(1+{ }^{8} C_{1}+{ }^{8} C_{2}\right)+\ldots \\
& \quad+\left(1+{ }^{n-1} C_{1}+{ }^{n-1} C_{2}\right) \\
= & 1+n \times 1+{ }^{1} C_{1}+{ }^{2} C_{1}+\ldots+{ }^{n-1} C_{1}+{ }^{2} C_{2}+{ }^{8} C_{2}+\ldots+{ }^{n-1} C_{2} \\
= & 1+{ }^{n} C_{1}+{ }^{n} C_{2}+{ }^{n} C_{3} \\
& \quad r C_{r}+{ }^{r+1} C_{r}+{ }^{r+2} C_{r}+\ldots+{ }^{n-1} C_{r}={ }^{n} C_{r+1} .
\end{aligned}
$$

- CaYley (Mese. Math., IV, p. 167) gives this formula in the form $\frac{1}{2}\left(n^{2}+n+2\right)$, and the oorresponding formula for $\phi_{3}(n), \frac{1}{8}\left(n^{2}+5 n+6\right)$. 
Again, with similar restrictions

$$
\begin{aligned}
\phi_{4}(n)= & 2+\phi_{3}(1)+\phi_{3}(2) \ldots+\phi_{3}(n-1) \\
= & 1+1+\left(1+{ }^{1} C_{1}\right)+\left(1+{ }^{2} C_{1}+{ }^{2} C_{2}\right)+\left(1+{ }^{3} C_{1}+{ }^{3} C_{2}+{ }^{3} C_{3}\right) \\
& \quad+\left(1+{ }^{4} C_{1}+{ }^{4} C_{2}+{ }^{4} C_{3}\right)+\ldots+\left(1+{ }^{n-1} C_{1}+{ }^{n-1} C_{2}+{ }^{n-1} C_{3}\right) \\
= & 1+n \times 1+{ }^{1} C_{1}+{ }^{2} C_{1} \ldots+{ }^{n-1} C_{1}+{ }^{2} C_{2}+{ }^{3} C_{2}+\ldots+{ }^{n-1} C_{2} \\
& +{ }^{3} C_{3}+{ }^{4} C_{3}+\ldots+{ }^{n-1} C_{3} \\
= & 1+{ }^{n} C_{1}+{ }^{n} C_{2}+{ }^{n} C_{3}+{ }^{n} C_{4} .
\end{aligned}
$$

This is easily extended by mathematical induction to the general case

$$
\phi_{p}(n)=1+{ }^{n} C_{1}+{ }^{n} C_{2}+{ }^{n} C_{3} \ldots+{ }^{n} C_{p}
$$

This formula remains true even if $p>n$, though in that case some of the terms towards the end of the series will be zero. The restrictions in this general case are that of the $n$ separating $S_{p-1}$ 's each pair intersects in a $S_{p-2}$, no 3 have a common $S_{p-2}$

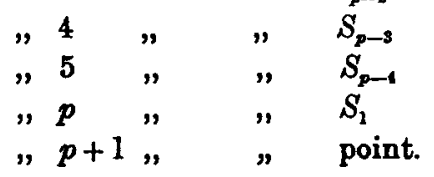

Cor. 1. $\phi_{n}(n)=1+{ }^{n} C_{1}+{ }^{n} C_{2} \ldots+{ }^{n} C_{n}=2^{n}$

Cor. 2. $\phi_{n+1}(n)=\phi(n)$

$$
\phi_{n+m}(n)=\phi(n)
$$

Cor. 3. $\phi_{p+1}(n)=\phi_{p}(n)+{ }^{n} C_{p+1}$, or $\phi_{p}(n)=\phi_{p-1}(n)+{ }^{n} C_{p}$

II.

Let now $C_{r}$ denote a finite unbounded closed space of $r$ dimensions, so that $C_{1}$ is a simple circuit, say the $\odot^{\text {ce }}$ of a circle, $C_{2}$ a spherical surface or other topically equivalent surface, $C_{3}$ a Riemann finite unbounded (elliptic) space of 3 dimensions, etc.; and let $\psi_{r}(n)$ be the number of separate regions into which a $C_{r}$ is divided by a set of $n C_{r-1}$ s such that each pair intersect in a $C_{r-2}$, but

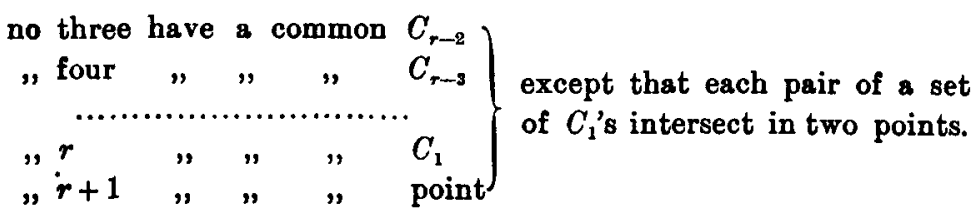

* A particular case of this Thenrem, viz., $\phi_{n}(n+1)=22_{n}+1-1$ is given in Schoute's MChrdimensionale Geometric, II (Leipzig 1905), 6. 
Then $\psi_{1}(n)=n={ }^{n} C_{1}$

$$
\begin{aligned}
\psi_{2}(1) & =2, \psi_{2}(2)=4 \psi_{2}(3)=\psi_{2}(2)+4 \quad \psi_{2}(n)=\psi_{3}(n-1)+2 \psi_{1}(n-1) \\
\psi_{2}(n) & =2(1+1+2+3 \ldots+n-1)=2\left(1+{ }^{n} C_{2}\right) \\
\psi_{3}(n) & =2+\psi_{2}(1)+\psi_{2}(2) \ldots+\psi_{2}(n-1) \\
& =2\left(1+1+1+{ }^{2} C_{2}+1+{ }^{3} C_{2} \ldots+1+{ }^{n-1} C_{2}\right) \\
& =2\left(n+{ }^{n} C_{3}\right) \\
\psi_{4}(n) & =2+\psi_{3}(1)+\psi_{3}(2) \ldots+\psi_{3}(n-1) \\
& =2\left(1+2+0+\left(3+{ }^{3} C_{3}\right)+\left(4+{ }^{4} C_{3}\right) \ldots+\left(n-1+{ }^{n-1} C_{3}\right)\right. \\
& =2+2\left({ }^{3} C_{1}+0\right)+2\left({ }^{3} C_{1}+0\right)+2\left({ }^{3} C_{1}+{ }^{3} C_{3}\right) \ldots+2\left({ }^{n-1} C_{1}+{ }^{n-1} C_{3}\right) \\
& =2\left(1+{ }^{n} C_{2}+{ }^{n} C_{4}\right)=2\left({ }^{n} C_{4}+{ }^{n} C_{2}+1\right) .
\end{aligned}
$$

Similarly,

$$
\begin{aligned}
& \psi_{3}(n)=2\left({ }^{n} C_{6}+{ }^{n} C_{3}+{ }^{n} C_{1}\right) \\
& \psi_{p}(n)=2\left({ }^{n} C_{p}+{ }^{n} C_{p-2}+{ }^{n} C_{p-4}+\ldots\right)
\end{aligned}
$$

where ${ }^{n} C_{0}$ means 1 .

Cor. 1. $\quad \psi_{r+1}(n)+\psi_{r}(n)=2\left({ }^{n} C_{r+1}+{ }^{n} C_{r}+{ }^{n} C_{r-1} \ldots+{ }^{n} C_{1}+1\right) \ldots(8)$

Cor. 2. $\psi_{n}(n)=2\left({ }^{n} C_{n}+{ }^{n} C_{n-3}+\ldots\right)$

$$
\psi_{n-1}(n)=2\left({ }^{n} C_{n-1}+{ }^{n} C_{n-3}+\ldots\right) \text {. }
$$

$\therefore \psi_{n}(n)+\psi_{n-1}(n)=2(1+1)^{n}=2_{1} 2^{n}$

and $\psi_{n}(n)-\psi_{n-1}(n)=2(1-1)^{n}=0$.

$\therefore \psi_{n}(n)=\psi_{n-1}(n)=2^{n}$,

e.g. $\psi_{2}(2)=2^{2}, \quad \psi_{1}(3)=3^{3}, \quad \psi_{3}(3)=\psi_{2}(3)=2^{3}$.

Cor. 3. $\psi_{n+m}(n)=\psi_{n}(n)=2^{n}$

\section{III.}

Let $\chi_{r}(n)$ denote the number of completely enclosed, nonoverlapping compartments which can be formed in a $S_{r}$ by $n$ flat $S_{r-1}$ 's each pair of which intersect in a $S_{r-2}$ under the restrictions

$$
\begin{aligned}
& \text { no three } S_{r-1} \text { 's have a common } S_{r-2} \\
& \text { "four } S_{r-1} \text { 's }, \quad " S_{r-3}
\end{aligned}
$$

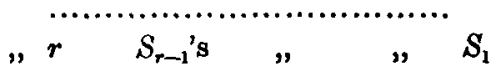

$$
\begin{aligned}
& " r+1 " \quad \text { " point. }
\end{aligned}
$$




\section{4}

$$
\begin{gathered}
\chi_{1}(1)=0, \quad \chi_{1}(2)=1, \quad \chi_{1}(3)=2 \ldots \chi_{1}(n)=n-1={ }^{n-1} C_{1} \\
\chi_{2}(1)=0, \quad \chi_{2}(2)-0, \quad \chi_{2}(3)-1, \quad \chi_{2}(4)=\chi_{2}(3)+\chi_{1}(3) \ldots \ldots \ldots \\
\chi_{2}(n)=\chi_{2}(n-1)+\chi_{1}(n-1) .
\end{gathered}
$$

Therefore $\chi_{2}(n)={ }^{n-2} C_{1}+{ }^{n-3} C_{1}+{ }^{n-4} C_{1} \ldots+{ }^{1} C_{1}={ }^{n-1} C_{2}$.

Similarly $\chi_{3}(n)={ }^{n-2} C_{2}+{ }^{n-3} C_{2} \quad . .+{ }^{2} C_{2}={ }^{n-1} C_{3}$.

And generally $\chi_{r}(n)={ }^{n-1} C_{r}$

when " $C_{r}$ is the number of combinations of $n$ things taken $r$ at a timo.

We had $\phi_{r}(n)=\phi_{r-1}(n)+{ }^{n} C_{r}$.

Therefore $\phi_{r}(n)=\phi_{r-1}(n)+\chi_{r}(n+1)$

Query: Can this be interpreted directly? 